\title{
O insight em Psiquiatria
}

\author{
Ana Margarida P. Cardoso ${ }^{\star}$
}

\begin{abstract}
Resumo
O sinal de que algo está a acontecer contribui para que o paciente reconheça que alguma coisa de estranho se está a passar consigo. Este reconhecimento faz com que o sujeito possa desempenhar uma função activa e seja um elemento colaborante do seu processo de recuperação. Cada doença apresenta, contudo, diferentes sintomas, uma vez que cada doença psiquiátrica consiste em diferentes perturbações com diversos efeitos sobre o funcionamento mental. Desta maneira, o fenómeno do insight que é registado em cada doença é diferente e expressa-se sob diferentes formas, não somente devido às manifestações clínicas da doença mas também devido às características individuais do sujeito.
\end{abstract}

Palavras-chave: insight; psicose; esquizofrenia.

\section{Insight IN Psychiatry}

\begin{abstract}
The sign that something is happening contribute for the patient recognize something strange is happening with him. The recognize do that the subject may perform a active function and be a collaborate element of their recovery process. Each disease presents, however, different symptoms, once that each psychiatric illness consists in different disorders with several effects under the mental function. This way, the insight phenomenon recorded in each illness is different and expressed under different ways, not only do to clinical manifestations of the illness but also to the subject individual characteristics.
\end{abstract}

Keywords: insight; psychosis; schizophrenia.

\footnotetext{
* Psicopedagoga e Mestre em Saúde Mental. Colaboradora na Faculdade de Ciências Médicas, Universidade Nova de Lisboa, Campo dos Mártires da Pátria, n. ${ }^{\circ}$ 130, 1169-056 Lisboa. Contacto: amargaridapc@gmail.com
} 


\subsection{O INSIGHT NAS PSICOSES}

É comum observar-se na prática clínica que grande parte das pessoas a quem foi diagnosticada uma psicose, podem não reconhecer a doença.

Uma vez que o impacto desta patologia é sentido em muitos aspectos da vida do indivíduo, o tratamento efectivo desta perturbação deve ser direccionado a vários níveis, inclusive em melhorar o insight da pessoa doente, para que, deste modo, se promova uma adesão ao tratamento e se possibilite uma intervenção precoce com vista à prevenção de recaídas, através da participação activa das pessoas com este diagnóstico.

A importância do insight, como este interage com a adesão e afecta o desfecho clínico nas perturbações psicóticas, é uma questão que tem envolvido muitos pesquisadores na área da saúde mental.

Alguns estudos têm sugerido que a falta de insight para a doença mental pode ser um factor preditivo importante no curso das perturbações psicóticas, principalmente em relação à adesão ao tratamento (AMADOR; STRAUSS, 1993).

Em psiquiatria, segundo Marková e Berrios (1992a), o termo insight refere-se a um particular estado de mente, evidenciado pelo conteúdo da fala e do comportamento. Segundo a autora, o principal problema que acompanha os estudos empíricos sobre o insight é a falta de definições consistentes e o método pelo qual os estudos foram avaliados. Este é um problema que dificulta conclusões válidas e comparações significativas entre os estudos.

Numa revisão efectuada sobre o conceito de insight, Marková e Berrios (1995a) acrescentam que, uma escassa atenção tem sido dada à base teórica em relação ao uso destes termos entre os diversos estudos. Consideram ser comum nas avaliações, que o investigador focalize um ou dois componentes do insight: reconhecimento da doença e reconhecimento da necessidade ou benefício do tratamento. Poucos estudos se têm preocupado em explorar a possibilidade de um conceito mais amplo para o insight, e a determinação da terminologia utilizada é decorrente de diferenças conceituais subjacentes. De um lado, a falta de insight é entendida como uma defesa psicológica e, no outro extremo, denota uma posição teórica implicando um déficit neurocognitivo (AMADOR; STRAUSS, 1993; FENTON; MCGLASHAN, 1994; YOUNG et al., 1998).

Marková e Berrios (1992) propuseram ainda uma complexidade maior quando conceptualizaram a consciência da doença como uma subcategoria de auto-conhecimento em vez de um ponto característico independente das perturbações psicóticas.

O insight é, sem dúvida, um fenómeno complexo e multidimensional. Na década passada, os investigadores aumentaram a sua atenção para a falta de $i n-$ sight como uma importante característica da esquizofrenia e também da doença bipolar. Enquanto que a etiologia desta falta de insight permanece pouco clara, 
alguns estudos recentes fortaleceram particularmente a hipótese dos défices neuropsicológicos estarem na origem de casos de insight muito fraco que persiste através do tempo.

À medida que as investigações vão decorrendo, começa-se a pintar um retrato cada vez mais claro sobre a natureza deste sintoma nas perturbações psicóticas, o que, consequentemente, vai permitir uma compreensão mais detalhada acerca deste fenómeno e o desenvolvimento de abordagens específicas que permitam ajudar as pessoas que não reconhecem a sua doença mental.

Uma coisa é certa, um não reconhecimento persistente e grave da doença pode transtornar não apenas a relação da pessoa afectada com aqueles que ama e com profissionais de saúde mental, como também as suas oportunidades de recuperarem e levarem vidas mais satisfatórias e produtivas. E por esta razão que as intervenções que pretendem melhorar directamente o insight ou perceber as consequências negativas de um fraco insight (não adesão, isolamento social, indiferença a pessoas que lhes são queridas e sistema de saúde) deverão ser desenvolvidas.

Devido à prevalência deste fenómeno e ao facto do mesmo interferir com o processo de recuperação da pessoa doente, este assunto tem despertado o interesse por parte de alguns investigadores, que se têm debruçado acerca da compreensão do insight nas psicoses.

\section{O IMPACTO DO INSIGHT NO TRATAMENTO DA ESQUIZOFRENIA}

Por a esquizofrenia representar uma doença com características tão heterogéneas, as abordagens multidisciplinares são fundamentais ao longo do processo de tratamento.

A doença deve ser considerada nos seus diversos aspectos e o tratamento deverá actuar a vários níveis para conseguir manter ou reconduzir, completa ou parcialmente, o indivíduo a uma qualidade de vida aceitável.

Actualmente, todos os especialistas são consensuais em considerar que a estratégia a ser utilizada na abordagem a esta doença deve partir de uma intervenção organizada segundo um programa que não é apenas de tipo farmacológico, mas de organização e assistência tanto à pessoa doente como à família.

O tratamento da esquizofrenia assenta assim em três componentes principais. Primeiro, há medicamentos para aliviar os sintomas e evitar a recidiva. Segundo, a educação e as intervenções psicossociais ajudam as pessoas doentes e os seus familiares a fazer face à doença e às suas complicações, bem como a evitar recorrências. Terceiro, a reabilitação ajuda as pessoas doentes a reintegrarem-se na comunidade e a recuperar o funcionamento social, educacional e ocupacional.

O tratamento farmacológico é fundamental, na medida em que permite obter melhores resultados, sobretudo quando combinado com a intervenção psicossocial que tende a minimizar o impacto de acontecimentos derivados do contexto 
em que a pessoa doente vive. Além disso, estudos realizados até à data, demonstraram que a combinação das diversas estratégias de tratamento também permite diminuir a ocorrência de recaídas.

Vários aspectos e características devem ser considerados para elaboração de um plano de tratamento para estas pessoas e, sempre que possível, deve-se envolver a família. A maioria dos pacientes necessita de cuidados ao longo da vida e estes visam, de modo geral, a diminuir a frequência, a gravidade e as consequências dos episódios psicóticos. Grande parte dos indivíduos, depois que adoecem, acabam por deixar de fazer as actividades que anteriormente vinham realizando, muitas vezes isolando-se das relações sociais e familiares. Muitos não têm qualquer actividade produtiva, necessitando de cuidados e amparo familiar definitivamente.

Recentemente, dentro do processo terapêutico da doença, tem havido um interesse cada vez maior no trabalho psicoeducativo, revelando bons resultados na evolução da doença com redução das taxas de recaída (GOLDSTEIN, 1994; LEFF, 1994; MACFARLANE et al., 1995).

A psicoeducação, no contexto do tratamento da esquizofrenia, é uma técnica que visa melhorar a compreensão da doença pelas pessoas doentes e suas famílias e, desta forma, melhorar o seu comportamento e atitude face à doença. Dentro deste contexto, o indivíduo adquire um papel activo no tratamento da sua doença. Procura-se assim, fornecer informação às pessoas doentes sobre vários aspectos da própria doença, de uma forma sistematizada, englobando áreas como, os sintomas da doença, as suas possíveis etiologias, o tratamento e o curso da mesma, procurando melhorar a adesão à terapêutica e o próprio insight da pessoa doente face à doença. A partir daí, visa-se a estimular as pessoas doentes e familiares a comprometerem-se activa e criticamente com o tratamento.

Actualmente, a psicoeducação tem representado uma importante abordagem psicossocial utilizada no tratamento da esquizofrenia, pois "informação clara e objectiva sobre a esquizofrenia é o primeiro passo para que se possa compreender e ajudar a pessoa doente e os seus familiares" (LOUZ ̃̃ NETO, 1996, p. 21).

\subsection{O ENVOLVIMENTO DA FAMÍLIA NO TRATAMENTO}

A noção de considerar também a família como unidade de tratamento é com efeito relativamente recente em saúde mental.

Em vários trabalhos realizados, verificou-se que a implementação de programas de intervenção familiar ajuda a reduzir o número de recaídas, melhorando ainda a adesão à terapêutica (MARI; STREINER, 1994).

De acordo com os estudos já efectuados, são vários os problemas que se deparam às famílias de pessoas com uma doença mental crónica (GRAHAM, 1983; TESSLER; KILLIAN; GUBMAN, 1987). Um desses problemas consiste, precisamente, nas dificuldades sentidas pelas famílias em lidar com a sintomatologia e comportamentos dos seus familiares doentes (ex: alucinações, ideias per- 
secutórias, perturbações do afecto, alterações do humor, alterações nos hábitos de comer e de dormir, comportamentos violentos, apatia, descuido com a higiene pessoal, etc.) (BIEGEL; SALES; SCHULZ, 1991; ST. ONGE; LAVOIE, 1987 apud CARPENTER et al., 1992).

O aparecimento de uma doença mental no seio de uma família, vem concerteza abalar o funcionamento e toda a dinâmica familiar, tendo em conta todo o estigma ainda existente em relação à doença mental.

A primeira referência sobre o sofrimento familiar aparece em 1946 com Treudley, que introduziu o conceito de "burden" na literatura psiquiátrica inglesa. É apenas no século XX que surge profissionalmente o objectivo de ajudar a família em encontrar soluções novas para os seus problemas internos e desta maneira diminuir as manifestações de sofrimento psicológico e responder às suas principais necessidades.

Por a esquizofrenia representar uma doença com características tão heterogéneas, as abordagens multiprofissionais são assim fundamentais para um programa de tratamento, o qual deve proporcionar uma resposta eficaz às necessidades expressas pelas pessoas doentes e respectivos familiares.

Segundo a American Psychiatic Association (2000, p. 886):

[...] os componentes específicos do tratamento com pessoas com esquizofrenia incluem: estabelecimento e manutenção de uma aliança terapêutica; monotorização do estado psiquiátrico do paciente; transmissão de educação com relação à doença e ao seu tratamento; determinação da necessidade de tratamento farmacológico e de terapias específicas; desenvolvimento de um plano geral de tratamento; melhora na adesão ao plano proposto; aumento do entendimento e adaptação aos efeitos psicossociais da doença; identificação e início precoce do tratamento de novos episódios; abordagem dos factores que precipitam e/ou perpetuam os episódios; abordagem familiar tanto para aliviar o sofrimento como melhorar o funcionamento familiar; facilitação do acesso a serviços e a recursos de saúde mental e outros sistemas relacionados.

\section{A importânCia do insight no tratamento}

Para que estes objectivos possam ser atingidos, é fundamental que as pessoas doentes adiram ao plano de tratamento. No entanto, é comum que estes utentes deixem de tomar a medicação e que frequentemente faltem às consultas, uma vez que, associado a este aspecto, se encontra a tal falta de insight destas pessoas face à sua doença mental.

$\mathrm{O}$ verdadeiro desafio na prestação de cuidados às pessoas que sofrem de esquizofrenia, está na necessidade de organizar serviços que garantam uma acção contínua, desde a identificação precoce até ao tratamento regular e à reabilitação. 
As metas dos cuidados consistem em identificar a doença o mais cedo possível, tratar os sintomas, conferir aptidões às pessoas doentes e aos seus familiares, manter a melhoria durante um certo período de tempo, evitar recaídas e reintegrar as pessoas com doença mental na comunidade, para que elas possam levar uma vida normal.

Ao longo da experiência profissional que tenho desenvolvido, numa enfermaria de doentes agudos de um hospital psiquiátrico (HOSPITAL MIGUEL BOMBARDA, UNIDADE DE INTERNAMENTO DE DOENTES AGUDOS DE LISBOA - UIAL), tenho verificado que a esquizofrenia representa a principal causa de internamento. É por isso importante aperfeiçoar, adequar e desenvolver metodologias de intervenção específicas durante a hospitalização.

As estruturas de internamento, especialmente as de internamento psiquiátrico, são aquelas em que as condições de um bom "ambiente terapêutico" devem ser equacionadas com maior cuidado - assim sendo, deve a "atmosfera" dessas estruturas ser o mais acolhedora possível de maneira a que a pessoa doente se sinta apoiada. Do mesmo modo, é fundamental oferecer os cuidados de saúde necessários, que visem ir ao encontro das principais necessidades do utente, e que dessa forma possam contribuir eficazmente para uma recuperação favorável que facilite o retorno ao ambiente familiar e social do sujeito, o mais depressa possível.

Actualmente, graças às terapias modernas, poucas pessoas com esquizofrenia necessitam de uma hospitalização prolongada. A duração média desse internamento pode variar entre duas a quatro semanas.

Tem sido estimado que cerca de $80 \%$ das recaídas são preveníveis (AMENSON, 1998) e nesse seguimento fará sentido que a reabilitação se focalize na prevenção da recaída, reduzindo o risco de (novas) hospitalizações.

É pois fundamental promover o empowerment das pessoas doentes mesmo durante a hospitalização, de forma a facilitar a participação activa na definição e implementação de medidas que favoreçam a sua autonomização.

O empowerment corresponde a um conjunto de estratégias de fortalecimento do poder, da autonomia e da auto-organização e é visto como um processo através do qual as pessoas ou as comunidades adquirem maior controlo sobre as decisões e acções que afectam a sua saúde (Health Promotion Glossary, Geneve: WHO, 1998).

O compromisso por parte da pessoa doente com o tratamento, e com a equipa de saúde que a acompanha, representa um factor fundamental para o seu melhor prognóstico. Seguindo correctamente o tratamento proposto, novas crises são evitadas e/ou minimizadas, impedindo-se assim o agravamento do quadro clínico.

Hoje em dia o termo adesão implica uma participação activa da pessoa doente no plano de tratamento e não um seguimento passivo, acrítico das instruções terapêuticas recebidas. 
Como o impacto da esquizofrenia é sentido em muitos aspectos da vida do indivíduo, o tratamento efectivo desta perturbação deve ser direccionado a vários níveis, inclusive em melhorar o insight da pessoa doente, para que deste modo se promova uma adesão ao tratamento e que consequentemente, possibilite uma intervenção precoce com vista à prevenção de recaídas, através da participação activa dos utentes com este diagnóstico.

\section{Conclusão}

A capacitação do utente para reconhecer aquilo que se passa consigo, deve ir para além da informação dada acerca da doença.

Pretende-se, a partir dessa compreensão, promover a qualidade de vida destas pessoas, através do desenvolvimento de novas aprendizagens que possibilitem uma cooperação activa, através do desenvolvimento das capacidade intactas, de maneira a possibilitar a aquisição de novos(s) comportamento(s) que tenham um impacte positivo nas queixas, sinais, sintomas, incapacidade e disfuncionalidade apresentados pelo/a utente, de maneira a que seja realizado um trabalho em conjunto entre o próprio e a equipa terapêutica. Sem essa associação e objectivo em comum, nenhum tratamento é realmente eficaz.

A importância do insight, como este interage com a adesão e afecta o desfecho clínico nas perturbações psicóticas, tem sido uma questão que tem envolvido muitos investigadores na área de saúde mental e que, actualmente, têm dedicado uma atenção especial ao estudo, avaliação e compreensão do fenómeno.

A natureza clínica, emocional e/ou intelectual do termo ajuda-nos a compreender a complexidade da dificuldade que existe na sua "tradução". Daí que as definições atribuídas ao conceito sejam muito distintas e variem consoante a formação teórica do autor/investigador.

Segundo a abordagem psicopedagógica, o termo insight deverá ir para além das definições clínicas tradicionais, que limitam o fenómeno em termos de consciência para a doença mental. Considero, segundo a minha perspectiva pessoal e profissional, que o insight deverá representar algo que inclua a perspectiva pessoal que cada indivíduo tem em relação àquilo que se passa consigo, e que vá para além da consciência doença.

Este processo de reconhecimento e de aceitação, deverá assim possibilitar uma compreensão mais aprofundada acerca da realidade interna e externa que envolve cada sujeito, permitindo que se vá ao encontro das suas resistências, necessidades e capacidades, possibilitando assim a plena integração desses factores. Isto porque, cada vez mais é fundamental destacar a importância do ponto de vista e das representações que cada utente faz acerca da sua realidade.

Também o pleno entendimento, por parte do técnico de saúde, das particularidades que envolvem cada situação/caso, permitirá concerteza com que sejam desenvolvidas abordagens terapêuticas mais eficazes, que facilitem a compreensão de cada sujeito e que, consequentemente, promovam a sua adesão ao tratamento. 
Através da promoção de um auto-conhecimento mais profundo, acerca de si mesmo bem como da sua realidade externa, procurar-se-á proporcionar a cada sujeito, um mergulho que facilite o envolvimento pleno daquilo que o rodeia (CARDOSO, 2008).

\section{REFERÊNCIAS}

AMADOR, X. F.; STRAUSS, D. H. Poor insight in schizophrenia. Special Issue: fifth annual New York State Office of Mental Health Research Conference. Psychiatric Quarterly, [S.1.], v. 64, n. 4, p. 305-318, 1993.

AMENSON, C. Schizophrenia: Family Education Methods. Pasadena: Pacific Clinics Institute, 1998.

AMERICAN PSYCHIATRY ASSOCIATION. Diagnostic and Statistical Manual IV-TR. Washington: American Psychiatry Publishing, 2000, 992p.

BIEGEL, D. E.; SALES, E.; SCHULZ R. Family caregiving in chronic illness. London: Sage, 1991.

CARPENTER, N. et al. Burden of care of families not living with young schizophrenic relatives. Hospital and Community Psychiatry, [S.1.], v. 43, p. 3843, 1992.

CARDOSO, A. M. Estudo sobre o insight em pessoas com psicose. 2008. Dissertação (Mestrado)-Faculdade de Ciências Médicas, Universidade Nova de Lisboa, Lisboa, 2008.

EMPOWERMENT. In: HEALTH Promotion Glossary. Geneve: WHO, 1998.

FENTON, W. S.; MCGLASHAN, T. H. Natural history of schizophrenia subtypes: positive and negative symptons and long-term course. Arch. Gen. Psychiatry, [S.1.], v. 48, p. 978-986, 1994.

GOLDSTEIN, M. J. Psychoeducational and family therapy in relapse prevention. Acta Psychiatrica Scandinavica, v. 89, suppl. 382, p. 54-57, 1994.

GRAHAM, R. W. Adult day care: how families of the dementia patient respond. Journal of Gerontological Nursing, [S.1.], v. 15, p. 27-31, 1983.

LEFF, J. Working with families of schizophrenic patients. British Journal of Psychiatry, v. 164, p. 71-76, 1994.

MACFARLANE, W. R. et al. Multiple-family groups and psychoeducation in the treatment of schizophrenia. Arch Gen Psychiatry, [S.1.], v. 52, n. 8, p. 679-687, 1995. 
MARI, J. J.; STREINER, D. An overview of family interventions and relapse on schizophrenia: meta-analysis of research findings. Psychological Medicine, [S.1.], v. 24, p. 565-578, 1994.

LOUZÃ NETO, M. R. Convivendo com a Esquizofrenia: um guia para pacientes e familiares. São Paulo: Lemos, 1996.

MARKOVA, I. S.; BERRIOS, G. E. The assessment of insight in clinical psychiatry: a new scale. Acta Psychiatrica Scandinavica, [S.1.], v. 86, n. 2, p. 159-164, 1992a.

. The meaning of insight in clinical psychiatry. British Journal of Psychiatry, [S.1.], v. 160, n. 6, p. 850-860, 1992 b.

. Insight in clinical psychiatry: a new model. Journal of Nervous and

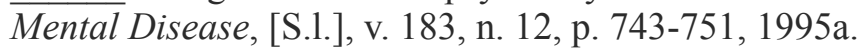

. Insight in clinical psychiatry revisited. Comprehensive Psychiatry, [S.1.], v. 36, n. 5, p. 367-376, 1995 b.

TESSLER, R. C.; KILLIAN, L. M.; GUBMAN, G. C. Stages in family response to mental illness: an ideal type. Psychosocial Reahabilitation Journal, [S.1.], v. 10, p. 3-16, 1987.

YOUNG, D. A. et al. Further parameters of insight and neuropsychological deficit in schizophrenia and other chronic mental disease. Journal of Nervous and Mental Disease, [S.1.], v. 186, n. 1, p. 44-50, 1998.

Recebido em: março de 2006

Aceito em: agosto de 2007 
